\title{
On symmetry, Lie symmetry and curved path particle motion: A case for hydrogen
}

\author{
JM Manale \\ The Department of Mathematical Sciences \\ University of South Africa \\ Florida, 1709, Johannesburg, Gauteng Province \\ Republic of South Africa \\ email:manaljm@unisa.ac.za, JacobManale@gmail.com
}

Received: January 6, 2021. Revised: March 12, 2021. Accepted: March 22, 2021. Published: March 29, 2021.

\begin{abstract}
We divert from popular practice by describing a motion of a macroscopic body, a hydrogen atom in this case, through quantum mechanics. What we realise is that a body can follow a curved path, without any external force acting on it, which is in contrast to Newtonian mechanics. To test the idea, we determine a formula for $G$, the universal gravitational constant.
\end{abstract}

Keywords- Dark matter and Dark energy; Gravitation; Schrödinger equation; Symmetry Analysis; Modified Symmetries.

\section{INTRODUCTION}

$\mathbf{I}$ SAAC Newton (1643-1727) proposed centuries ago, in what has become known as his first law of motion, that a body will remain at rest, or remain in a state of motion, in a straight line, unless acted upon by an external unbalanced force. We disagree.

Our view is that a body can follow a curved path, without any force acting on it, and the evidence thereof is in plain sight. One example is the gyroscope. The other is the concept upon which boomerangs operate by. One other is a trick skilful soccer players use to confuse opponents. What we do here is explain the common underlying motion through quantum super-positions and entanglements. This we do by solving the two-dimensional Schrödinger equation for a hydrogen atom, because of its simplicity: Not many particles are involved. We choose the two-dimensional space, because curve of motion is in a plane.

We had hoped to solve the equations through Sophus Lie's symmetry group theoretical methods, unfortunately the pure approach does not allow for super-positions. To circumvent this, we introduce what we call modified Lie symmetries, or simply modified symmetries. We use two concepts to evaluate the resulting integrals. The first makes use of intermediate value theorems, and the second is on differentiable topological manifolds.

\section{THE GORVENING EQUATIONS OF MOTION}

The Schrödinger wave equation for the hydrogen atom with electron mass $m_{e}$ and proton mass $m_{p}$ has the form

$$
\begin{aligned}
& -\frac{\hbar^{2}}{2 m_{e}}\left[\frac{\partial^{2}}{\partial x^{2}}+\frac{\partial^{2}}{\partial y^{2}}+\frac{\partial^{2}}{\partial z^{2}}\right] \psi \\
& -\frac{\hbar^{2}}{2 m_{p}}\left[\frac{\partial^{2}}{\partial X^{2}}+\frac{\partial^{2}}{\partial Y^{2}}+\frac{\partial^{2}}{\partial Z^{2}}\right] \psi \\
& +(V-E) \psi=i \hbar \frac{\partial \psi}{\partial t} .
\end{aligned}
$$

Here $\psi=\psi(t, \mathbf{r}, \mathbf{R})$ is the probability density function, $V$ is the potential, $E$ is the energy level, $\mathbf{r}=(x, y, z)$ is the position of the electron, $\mathbf{R}=(X, Y, Z)$ is that of the proton, and $\hbar$ is the reduced Planck constant.

Our interest is in the motion of the electron and proton on the same plane. This reduces (1) to the two-dimensional Schrödinger wave equation,

$$
\begin{aligned}
& -\frac{\hbar^{2}}{2 m_{e}}\left[\frac{\partial^{2}}{\partial x^{2}}+\frac{\partial^{2}}{\partial y^{2}}\right] \psi-\frac{\hbar^{2}}{2 m_{p}}\left[\frac{\partial^{2}}{\partial X^{2}}+\frac{\partial^{2}}{\partial Y^{2}}\right] \psi \\
& +(V-E) \psi=i \hbar \frac{\partial \psi}{\partial t}
\end{aligned}
$$

Here $\psi=\psi(t, \mathbf{r}, \mathbf{R})$ is the probability density function, $V$ is the potential, $E$ is the energy level, $\mathbf{r}=(x, y)$ is the position of the electron, $\mathbf{R}=(X, Y)$ is that of the proton.

Since the mass of the proton is much larger than that of the proton, then the electron can be interpreted as moving around around a positive core frozen in plavein the static potential field $V(R, r)$. This then splits (2) into

$$
-\frac{\hbar^{2}}{2 m_{p}}\left[\frac{\partial^{2}}{\partial X^{2}}+\frac{\partial^{2}}{\partial Y^{2}}\right] \varphi=i \hbar \frac{\partial \varphi}{\partial t}
$$

and

$$
-\frac{\hbar^{2}}{2 m_{e}}\left[\frac{\partial^{2}}{\partial x^{2}}+\frac{\partial^{2}}{\partial y^{2}}\right] \phi+(V-E) \phi=0,
$$

where $\psi=\varphi(t, \mathbf{R}) \phi(t, \mathbf{r})$. Equation (3) describes the motion of a free particle, implying that the proton is moving freely. Equation (4), on the other hand, is that of a forced particle. A transition to polar coordinates of (3), wherein $x=\rho \cos \theta$ and $y=\rho \sin \theta$, gives

$$
-\frac{\hbar^{2}}{2 m_{p}}\left[\frac{1}{\rho_{p}} \frac{\partial}{\partial \rho_{p}}\left(\rho \frac{\partial}{\partial \rho_{p}}\right)+\frac{1}{\rho_{p}^{2}} \frac{\partial^{2}}{\partial \theta_{p}^{2}}\right] \varphi_{p}=i \hbar \frac{\partial \varphi_{p}}{\partial t},
$$


or

$$
\frac{1}{\rho_{p}} \frac{\partial}{\partial \rho_{p}}\left(\rho \frac{\partial \varphi}{\partial \rho_{p}}\right)+\frac{1}{\rho_{p}^{2}} \frac{\partial^{2} \varphi_{p}}{\partial \theta_{p}^{2}}=\frac{2 m_{p}}{i \hbar} \frac{\partial \varphi}{\partial t} .
$$

The substitution $\varphi(t, \rho, \theta)=P(t, \rho) \Theta(t, \theta)$ leads to

$$
\frac{1}{\rho_{p}} \frac{d}{d \rho_{p}}\left(\rho \frac{d P_{p}}{d \rho_{p}}\right)+\left(\frac{2 m_{p}}{\hbar^{2}} E-\frac{\lambda_{p}}{\rho_{p}^{2}}\right) P_{p}=0
$$

and

$$
\frac{d^{2} \Theta_{p}}{\partial \theta_{p}^{2}}+\lambda_{p} \Theta_{p}=0
$$

Equation (4) separates into

$$
\frac{1}{\rho_{e}} \frac{d}{d \rho_{e}}\left(\rho_{e} \frac{d P_{e}}{d \rho_{e}}\right)-\left(\frac{2 m_{e}}{\hbar^{2}}(V-2 E)-\frac{\lambda_{e}}{\rho_{e}^{2}}\right) P_{e}=0
$$

and

$$
\frac{d^{2} \Theta_{e}}{\partial \theta_{e}^{2}}+\lambda_{e} \Theta_{e}=0
$$

It is assumed that $\partial \phi_{e} / \partial t=-i E \phi_{e} / \hbar$. In the case of a coulombic force, we have

$$
V=-\frac{1}{4 \pi \epsilon_{0}} \frac{q Q}{\rho_{e}}
$$

where $\epsilon_{0}$ is the permittivity of free space, $q$ and $Q$ are charges.

\section{SOlVING (3), THE FREE PARTICLE WAVE EQUATION}

Being able to solve the Schrödinger equation exactly, is the key to particle states, their superpositions and entanglements. These assist in the transition from classical to quantum computing; allowing space for faster computers.

However, there are beliefs that this equation can be solved exactly only for the simplest of systems, such as the particle in a box problem, the Hydrogen atoms and the free particle, and not for complex systems. While we may agree with Toli and Zou [1] that that is the case and that multi-electron systems may probably be unsolvable, we argue here that even the simplest case, the free particle, was not solved correctly.

As a precursor to exact solutions, we begin by first pointing out the mis-steps. In literature, (3) is presented in the form

$$
\frac{\partial u}{\partial \zeta}=\frac{i}{2}\left(\frac{\partial^{2} u}{\partial R^{2}}+\frac{1}{R} \frac{\partial u}{\partial R}+\frac{1}{R^{2}} \frac{\partial^{2} u}{\partial \varphi^{2}}+\frac{\partial^{2} u}{\partial T^{2}}\right),
$$

and called the Airy-Laguerre-Gaussian equation. It models the propagation of energy packets in free space. Here $u=$ $u(\zeta, R, \varphi, T)$ is the complex envelope of the optical field, $R=$ $\sqrt{X^{2}+Y^{2}}=r / r_{0}$ is the dimensionless spatial coordinate, $T=t / t_{0}$ the retarded time, $\zeta=z /\left(k r_{0}^{2}\right)$ is the longitudinal propagation distance , $k=2 \pi N / \lambda_{0}$ is the wave number, with the wavelength $\lambda_{0}$, refractive index $N$ and $\varphi$ the azimuthal angle. The quantities $r_{0}$ and $t_{0}$ are scaling parameters.

Letting

$$
u(\zeta, R, \varphi, T)=V(\zeta, R) \Phi(\varphi) P(\zeta, T),
$$

separates (12) into three simpler components. This has been executed before, see [2], [3], [4], [5], [6], [7] and [8]. The simplest is the azimuthal equation,

$$
\frac{d^{2} \Phi}{d \varphi^{2}}+\lambda \Phi=0
$$

The parameter $m=\sqrt{\lambda}$ is called the azimuthal mode number, also known as the topological charge. Next is the 1D Schrödinger equation,

$$
\frac{\partial P}{\partial \zeta}-\frac{i}{2} \frac{\partial^{2} P}{\partial T^{2}}=0,
$$

and the Laguerre-Scholes equation,

$$
\frac{\partial V}{\partial \zeta}-\frac{i}{2}\left(\frac{\partial^{2} V}{\partial R^{2}}+\frac{1}{R} \frac{\partial V}{\partial R}-\frac{m^{2}}{R^{2}} V\right)=0 .
$$

It is thought that the azimuthal equation is solved by letting $\phi=\exp (\lambda \varphi)$. This gives $\phi=A \exp (-i \varphi)+B \exp (i \varphi)$, with $A$ and $B$ as constants. This solution is incorrect.

\section{SOlVING (4), THE FORCED WAVE EQUATION}

Letting $\varphi=\varpi(\mathbf{r}) \exp (i E t / \hbar)$ reduces (4) to

$$
-\frac{\hbar^{2}}{2 m_{e}}\left[\frac{\partial^{2}}{\partial x^{2}}+\frac{\partial^{2}}{\partial y^{2}}+\frac{\partial^{2}}{\partial z^{2}}\right] \varpi+(V-2 E) \varpi=0,
$$

a time independent Schrödinger equation.

The substitution $\varpi=R(r) \Theta(\theta) \Phi(\phi)$ reduces it to

$$
\frac{d^{2} \Theta}{d \theta^{2}}=m_{l}^{2} \Theta
$$

$$
\frac{1}{2 m r^{2}} \frac{d}{d r}\left(r^{2} \frac{d R}{d r}\right)-\left(V-2 E-\frac{l(l+1)}{2 m r^{2}}\right) R=0 .
$$

In the case of a coulombic force, we have

$$
V=-\frac{1}{4 \pi \epsilon_{0}} \frac{q Q}{r},
$$

so that

$$
\frac{1}{2 m r^{2}} \frac{d}{d r}\left(r^{2} \frac{d R}{d r}\right)+\left(\frac{1}{4 \pi \epsilon_{0}} \frac{q Q}{r}+2 E+\frac{l(l+1)}{2 m r^{2}}\right) R=0,
$$

where $\epsilon_{0}$ is the permittivity of free space, $q$ and $Q$ are charges, $r=\|\mathbf{r}\|$ is the relative distance and the position $\mathbf{s}$ of the hydrogen atom is given by its centre of mass:

$$
\mathbf{s}=\frac{m_{e} \mathbf{r}+m_{p} \mathbf{R}}{m_{e}+m_{p}} .
$$

The third equation is

$$
\frac{1}{\sin \theta} \frac{d}{d \theta}\left(\sin \theta \frac{d \Theta}{d \theta}\right)-\left(l(l+1)-\frac{m_{l}^{2}}{\sin ^{2} \theta}\right) \Theta=0 .
$$

\section{A. Solutions through differentiable Manifolds}

\section{CONCLUSION}

Newtonian mechanics has led to great results and a vast number of technological applications. Unfortunately, a number of confusing concepts have also resulted. Dark matter and energy is one of such concepts. In our view, this results when people have absolute belief and trust in a theory. Our suggestion that motion along a curved path is possible without an external force, will greatly assist in addressing the confusion. 


\section{REFERENCES}

[1] Ilia Toli and Shengli Zou. Schrdinger equation with coulomb potential admits no exact solutions. Chemical Physics Letters: X, 2, 42019.

[2] Zhenkun Wu, Zhiping Wang, Hao Guo, Wei Wang, and Yuzong Gu. Self-accelerating airy-laguerre-gaussian light bullets in a two-dimensional strongly nonlocal nonlinear medium. Optics Express, 25:30468, 112017.

[3] Yixian Qian and Site Zhang. Quasi-airy beams along tunable propagation trajectories and directions. Optics Express, 24:9489, 52016.

[4] Miao Dong, XingYuan Lu, Chengliang Zhao, Yangjian Cai, and Yuanjie Yang. Measuring topological charge of partially coherent elegant laguerregaussian beam. Optics Express, 26:033807-1-033806-6, 122018.

[5] Lifu Zhang, Kun Liu, Haizhe Zhong, Jinggui Zhang, Ying Li, and Dianyuan Fan. Effect of initial frequency chirp on airy pulse propagation in an optical fiber. Optics Express, 23:2566, 22015.

[6] Dawoon Choi, Kyookeun Lee, Keehoon Hong, Il-Min Lee, Kyoung-Youm Kim, and Byoungho Lee. Generation of finite power airy beams via initial field modulation. Optics Express, 21:18797, 82013.

[7] Wei-Ping Zhong, Milivoj Beli, and Yiqi Zhang. Three-dimensional localized airy-laguerre-gaussian wave packets in free space. Optics Express, 23:23867, 92015.

[8] A. V. Volyar and T. A. Fadeeva. Laguerre-gaussian beams with complex and real arguments in a uniaxial crystal. Optics and Spectroscopy (English translation of Optika i Spektroskopiya), 101:450-457, 92006.

\section{Creative Commons Attribution License 4.0 (Attribution 4.0 International, CC BY 4.0)}

This article is published under the terms of the Creative Commons Attribution License 4.0

https://creativecommons.org/licenses/by/4.0/deed.en_US 